\title{
Human activities detection and classification based on micro-Doppler signatures near the baseline of forward scattering radar
}

\begin{abstract}
Fall poses a major problem, which raises the concern of elderly populations aged 65 and above in all over the world. In this paper, we propose Forward Scattering Radar system as a Doppler sensor in distinguishing features of fall events from non-fall activities. The signal features of joint time-frequency representations are used for detection, while the support vector machine, which is based on the short-time Fourier transform feature, has been used in the classification process. An indoor experiment was conducted to emulate the elderly people's daily activities and the falling down event, where 50 trials were carried out by five adults for each of the activity. The detection results indicated that the forward scattering radar has a high ability in detecting the micro-Doppler signatures generated from the low speed motion of a human body segments during daily activities. The preliminary classification results are $100 \%$ for the corresponding free fall-sitting on a chair, free fall-sitting on the floor, and for all three activities.
\end{abstract}

Keyword: Elderly fall detection; Forward scattering radar; Time-frequency domain analysis; Support vector machine; Classification 